PROCEEDINGS OF THE

AMERICAN MATHEMATICAL SOCIETY

Volume 125, Number 12, December 1997, Pages 3561-3567

S 0002-9939(97)04091-4

\title{
DIFFERENTIAL OPERATORS HAVING SOBOLEV TYPE LAGUERRE POLYNOMIALS AS EIGENFUNCTIONS
}

\author{
H. BAVINCK
}

(Communicated by Christopher D. Sogge)

Abstract. We consider the polynomials $\left\{L_{n}^{\alpha, M}(x, l)\right\}_{n=0}^{\infty}$ orthogonal with respect to the Sobolev type inner product

$$
\langle p, q\rangle=\frac{1}{\Gamma(\alpha+1)} \int_{0}^{\infty} p(x) q(x) x^{\alpha} e^{-x} d x+M p^{(l)}(0) q^{(l)}(0),
$$

where $\alpha>-1, M \geq 0$ and $l$ is a nonnegative integer. It is the purpose of this paper to show that these polynomials are eigenfunctions of a class of linear differential operators containing one that is of finite order $2 \alpha+4 l+4$ if $\alpha$ is a nonnegative integer and $M>0$.

\section{INTRODUCTION}

We study linear differential operators for which the polynomials $\left\{L_{n}^{\alpha, M}(x, l)\right\}_{n=0}^{\infty}$ orthogonal with respect to the Sobolev type inner product

$$
\langle p, q\rangle=\frac{1}{\Gamma(\alpha+1)} \int_{0}^{\infty} p(x) q(x) x^{\alpha} e^{-x} d x+M p^{(l)}(0) q^{(l)}(0),
$$

where $p$ and $q$ are polynomials, $\alpha>-1, M \geq 0$ and $l$ is a nonnegative integer, are eigenfunctions. This inner product is a special case of the general inner product considered by R. Koekoek in his thesis [12] (see also [11]),

$$
\langle p, q\rangle=\frac{1}{\Gamma(\alpha+1)} \int_{0}^{\infty} p(x) q(x) x^{\alpha} e^{-x} d x+\sum_{k=0}^{l} M_{k} p^{(k)}(0) q^{(k)}(0),
$$

$\alpha>-1, M_{k} \geq 0$ for $k=0,1, \cdots, l$ and $l$ is a nonnegative integer. For the inner product (1) in the case $l=0$ the orthogonal polynomials $L_{n}^{\alpha, M}(x)=L_{n}^{\alpha, M}(x, 0)$ have been introduced by Koornwinder [13] (for $\alpha \in\{0,1,2\}$ they had been introduced earlier, see [7], [5], [8], [6]) and for these polynomials J. and R. Koekoek [9] (see also [1]) showed that they are eigenfunctions of a differential operator, linear in $M$, which in the case $M>0$ is of finite order $2 \alpha+4$ if $\alpha$ is a nonnegative integer and is of infinite order for other values of $\alpha$. A first generalization of this important result is found in [10], where the polynomials $\left\{L_{n}^{\alpha, M, N}(x)\right\}_{n=0}^{\infty}$ orthogonal with respect

Received by the editors June 27, 1996.

1991 Mathematics Subject Classification. Primary 33C45, 34A35.

Key words and phrases. Differential operators, Sobolev type Laguerre polynomials.

(C)1997 American Mathematical Society 
to the Sobolev type inner product

$$
\langle p, q\rangle=\frac{1}{\Gamma(\alpha+1)} \int_{0}^{\infty} p(x) q(x) x^{\alpha} e^{-x} d x+M p(0) q(0)+N p^{\prime}(0) q^{\prime}(0)
$$

were considered. After laborious calculations the authors succeeded in proving that these polynomials are eigenfunctions of a class of differential operators containing one which in the case that $\alpha$ is a nonnegative integer is of finite order. This order is $2 \alpha+4$ if $M>0, N=0$, is $2 \alpha+8$ if $M=0, N>0$ and finally is $4 \alpha+10$ if $M>0, N>0$.

In this paper we find another generalization of the main result of [9] by showing that the polynomials $\left\{L_{n}^{\alpha, M}(x, l)\right\}_{n=0}^{\infty}$, orthogonal with respect to (1), are eigenfunctions of a class of differential operators linear in $M$ containing one which in the case that $\alpha$ is a nonnegative integer is of finite order $2 \alpha+4+4 l$, if $M>0$. The cases $l=0$ and $l=1$ were already known from the preceding results.

The most remarkable thing in this paper is perhaps not the result itself, which could be predicted, but the relatively easy way it is obtained, compared to the foregoing papers. This is due to two facts:

a) By the results obtained in [4] and [2] the existence of differential operators linear in $M$, possibly of infinite order, having the polynomials $\left\{L_{n}^{\alpha, M}(x, l)\right\}_{n=0}^{\infty}$ as eigenfunctions is assured and a formula for the eigenvalues is known.

b) In [3] the formula

$$
\sum_{j=k}^{i} j^{s} L_{i-j}^{(-\alpha-i-1)}(-x) L_{j-k}^{(\alpha+k)}(x)=\delta_{i, 2 s+k}(-x)^{s}, i, k, s \in\{0,1,2, \cdots\}
$$

for all $x, \alpha \in \mathbb{C}$ provided that $i \geq 2 s+k$ has been deduced which appears to be a powerful tool for showing that the differential operator is of finite order if $\alpha$ is a nonnegative integer.

\section{Representation of the POLYNOMIALS}

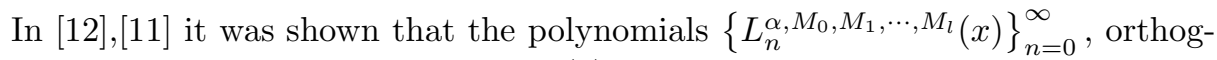
onal with respect to the inner product (2), can be put in the form

$$
L_{n}^{\alpha, M_{0}, M_{1}, \cdots, M_{l}}(x)=\sum_{k=0}^{l+1} A_{n, k} D^{k} L_{n}^{(\alpha)}(x)
$$

$\left(D=\frac{d}{d x}\right)$ and the coefficients $\left\{A_{n, k}\right\}_{k=0}^{l+1}$ satisfy the equations

$$
\begin{gathered}
\left(\begin{array}{c}
m+\alpha \\
m
\end{array}\right) \sum_{k=m+1}^{l+1}(-1)^{k}\left(\begin{array}{l}
n-m-1 \\
k-m-1
\end{array}\right) A_{n, k} \\
+(-1)^{m} M_{m} \sum_{k=0}^{l+1}(-1)^{k}\left(\begin{array}{c}
n+\alpha \\
n-m-k
\end{array}\right) A_{n, k}=0, m=0,1,2, \cdots, l .
\end{gathered}
$$

In our special case these equations become

$$
\sum_{k=m+1}^{l+1}(-1)^{k}\left(\begin{array}{l}
n-m-1 \\
k-m-1
\end{array}\right) A_{n, k}=0, m=0,1,2, \cdots, l-1,
$$




$$
\left(\begin{array}{c}
l+\alpha \\
l
\end{array}\right) A_{n, l+1}=M \sum_{k=0}^{l+1}(-1)^{k}\left(\begin{array}{c}
n+\alpha \\
n-l-k
\end{array}\right) A_{n, k} .
$$

Lemma 2.1. For all real $n$ and all $m, l \in\{0,1, \cdots\}$ with $m \leq l$

$$
\sum_{k=m+1}^{l+1}(-1)^{l-k+1}\left(\begin{array}{l}
n-m-1 \\
k-m-1
\end{array}\right)\left(\begin{array}{c}
n-k \\
l-k+1
\end{array}\right)=\delta_{l, m} .
$$

Proof. If we write $s=k-m-1, t=l-m$ and $N=n-l-1$ then (6) reads

$$
\sum_{s=0}^{t}(-1)^{t-s}\left(\begin{array}{c}
N+t \\
s
\end{array}\right)\left(\begin{array}{c}
N+t-s \\
t-s
\end{array}\right)=\delta_{t, 0} .
$$

If we note that $\left(\begin{array}{c}N+t-s \\ t-s\end{array}\right)=(-1)^{t-s}\left(\begin{array}{c}-N-1 \\ t-s\end{array}\right)$, this follows by comparing the coefficient of $x^{t}$ at both sides of the identity

$$
(1+x)^{t-1}=(1+x)^{N+t}(1+x)^{-N-1} .
$$

From the lemma and (4) it follows that

$$
A_{n, k}=\left(\begin{array}{c}
n-k \\
l-k+1
\end{array}\right) A_{n, l+1} \text { for } k=1,2, \cdots, l+1
$$

and (5) yields

$$
\left(\begin{array}{c}
l+\alpha \\
l
\end{array}\right) A_{n, l+1}=M\left(\begin{array}{c}
n+\alpha \\
n-l
\end{array}\right) A_{n, 0}+M A_{n, l+1} \sum_{k=1}^{l+1}(-1)^{k}\left(\begin{array}{c}
n+\alpha \\
n-l-k
\end{array}\right)\left(\begin{array}{c}
n-k \\
l-k+1
\end{array}\right) .
$$

If the normalization of the orthogonal polynomials $L_{n}^{\alpha, M}(x, l)$ is chosen such that

$$
L_{n}^{\alpha, M}(x, l)=L_{n}^{(\alpha)}(x)+M Q_{n}^{\alpha, l}(x),
$$

then we obtain

$$
A_{n, k}=M\left(\begin{array}{c}
n-k \\
l-k+1
\end{array}\right) \frac{\left(\begin{array}{c}
n+\alpha \\
n-l
\end{array}\right)}{\left(\begin{array}{c}
l+\alpha \\
l
\end{array}\right)} \text { for } k=1,2, \cdots, l+1,
$$

and

$$
A_{n, 0}=1+\frac{M}{\left(\begin{array}{c}
l+\alpha \\
l
\end{array}\right)} \sum_{k=1}^{l+1}(-1)^{k-1}\left(\begin{array}{c}
n+\alpha \\
n-l-k
\end{array}\right)\left(\begin{array}{c}
n-k \\
l-k+1
\end{array}\right) .
$$

Hence

$$
\begin{gathered}
\left(\begin{array}{c}
l+\alpha \\
l
\end{array}\right) Q_{n}^{\alpha, l}(x)=\sum_{k=1}^{l+1}(-1)^{k-1}\left(\begin{array}{c}
n+\alpha \\
n-l-k
\end{array}\right)\left(\begin{array}{c}
n-k \\
l-k+1
\end{array}\right) L_{n}^{(\alpha)}(x) \\
-\left(\begin{array}{c}
n+\alpha \\
n-l
\end{array}\right) \sum_{k=1}^{l+1}(-1)^{k-1}\left(\begin{array}{c}
n-k \\
l-k+1
\end{array}\right) L_{n-k}^{(\alpha+k)}(x) .
\end{gathered}
$$

Another representation of the polynomials $Q_{n}^{\alpha, l}(x)$ is (see [14], specified here for the case of Laguerre polynomials)

$$
Q_{n}^{\alpha, l}(x)=\sum_{k=l}^{n} q_{n, k}^{\alpha, l} L_{k}^{(\alpha)}(x), n \in\{l+1, l+2, \cdots\},
$$


where

$$
q_{n, n}^{\alpha, l}=\sum_{k=l}^{n-1} \frac{\left(L_{k-l}^{(\alpha+l)}(0)\right)^{2}}{\left(\begin{array}{c}
k+\alpha \\
k
\end{array}\right)}=\frac{\Gamma(\alpha+1)}{(\Gamma(l+\alpha+1))^{2}} \sum_{k=l}^{n-1} \frac{\Gamma(k+\alpha+1) k !}{((k-l) !)^{2}}
$$

and for $n>k \geq l$

$$
q_{n, k}^{\alpha, l}=-\frac{\left(\begin{array}{c}
n+\alpha \\
n-l
\end{array}\right)\left(\begin{array}{c}
k+\alpha \\
k-l
\end{array}\right)}{\left(\begin{array}{c}
k+\alpha \\
k
\end{array}\right)}=-\left(\begin{array}{c}
n+\alpha \\
n-l
\end{array}\right) \frac{\Gamma(\alpha+1) k !}{\Gamma(l+\alpha+1)(k-l) !} .
$$

We take $Q_{n}^{\alpha, l}(x)=0$ if $n \leq l$.

\section{Differential operators}

We study differential operators of the form $\mathbf{L}^{\alpha}+M \mathbf{A}^{(\alpha, l)}$ having the polynomials $L_{n}^{\alpha, M}(x, l)$ as eigenfunctions with eigenvalues $\lambda_{n}+M \alpha_{n}^{(\alpha, l)}$ (see [2]), where here $\lambda_{n}=n$ and $\alpha_{0}=\alpha_{0}^{(\alpha, l)}=0$,

$$
\mathbf{L}^{\alpha}=-x D^{2}-(\alpha+1-x) D
$$

and the operator $\mathbf{A}^{(\alpha, l)}$ is of the form

$$
\mathbf{A}^{(\alpha, l)}=\sum_{i=1}^{\infty} a_{i}(x ; \alpha, l) D^{i}
$$

If $l \geq 1$ the eigenvalues $\alpha_{1}^{(\alpha, l)}, \alpha_{2}^{(\alpha, l)}, \cdots, \alpha_{l}^{(\alpha, l)}$ can be chosen arbitrarily. Let $\alpha_{k}^{(\alpha, l)}=$ $\alpha_{k}$ for $k=1,2, \cdots, l$. Then for $n>l$ (see [2])

$$
\begin{gathered}
\alpha_{n}^{(\alpha, l)=\alpha_{l}}+\sum_{m=l}^{n-1} q_{m+1, m+1}^{\alpha, l}=\alpha_{l}+\sum_{m=l}^{n-1} \frac{\Gamma(\alpha+1)}{(\Gamma(l+\alpha+1))^{2}} \sum_{k=l}^{m} \frac{\Gamma(k+\alpha+1) k !}{((k-l) !)^{2}} \\
=\alpha_{l}+\frac{\Gamma(\alpha+1)}{(\Gamma(l+\alpha+1))^{2}} \sum_{k=l}^{n-1} \frac{\Gamma(k+\alpha+1) k !(n-k)}{((k-l) !)^{2}} .
\end{gathered}
$$

The operator $\mathbf{A}^{(\alpha, l)}$ can be obtained by [2], formula (5),

$$
\left(\mathbf{L}^{\alpha}-n \mathbf{I}\right) Q_{n}^{\alpha, l}(x)+\left(\mathbf{A}^{(\alpha, l)}-\alpha_{n}^{(\alpha, l)} \mathbf{I}\right) L_{n}^{(\alpha)}(x)=0, n=0,1, \cdots,
$$

where $\mathbf{I}$ is the identity operator. Now

$$
\begin{aligned}
\left(\mathbf{L}^{\alpha}-n \mathbf{I}\right) L_{n-k}^{(\alpha+k)}(x) & =(n-k) L_{n-k}^{(\alpha+k)}(x)-k L_{n-k-1}^{(\alpha+k+1)}(x)-n L_{n-k}^{(\alpha+k)}(x) \\
= & -k\left(L_{n-k}^{(\alpha+k)}(x)+L_{n-k-1}^{(\alpha+k+1)}(x)\right) .
\end{aligned}
$$

Thus for $n=0,1, \cdots$

$$
\begin{gathered}
\left(\mathbf{A}^{(\alpha, l)}-\alpha_{n}^{(\alpha, l)} \mathbf{I}\right) L_{n}^{(\alpha)}(x)= \\
\frac{\left(\begin{array}{c}
n+\alpha \\
n-l
\end{array}\right)}{\left(\begin{array}{c}
l+\alpha \\
l
\end{array}\right)} \sum_{k=1}^{l+1}(-1)^{k}\left(\begin{array}{c}
n-k \\
l-k+1
\end{array}\right) k\left(L_{n-k}^{(\alpha+k)}(x)+L_{n-k-1}^{(\alpha+k+1)}(x)\right) .
\end{gathered}
$$

Note that for $n \leq l$ the right side vanishes. If in the case that $l \geq 1$ we put

$$
\mathbf{A}^{(\alpha, l)}=\mathbf{B}^{(\alpha, l)}+\sum_{k=1}^{l-1} \alpha_{k} \mathbf{J}^{(\alpha, k)}+\alpha_{l} \mathbf{K}^{(\alpha, l)}
$$


such that

$$
\mathbf{B}^{(\alpha, l)}=\sum_{i=1}^{\infty} b_{i}(x ; \alpha, l) D^{i}
$$

the operators $\mathbf{J}^{(\alpha, k)}$ of the form

$$
\mathbf{J}^{(a, k)}=\sum_{i=1}^{\infty} \gamma_{i}(x ; \alpha, k) D^{i}
$$

are defined by

$$
\mathbf{J}^{(\alpha, k)} L_{n}^{(\alpha)}(x)=\delta_{n, k} L_{n}^{(\alpha)}(x), n=0,1, \cdots,
$$

and the operator $\mathbf{K}$ of the form

$$
\mathbf{K}^{(\alpha, l)}=\sum_{i=1}^{\infty} \kappa_{i}(x ; \alpha, l) D^{i}
$$

is defined by

$$
\begin{gathered}
\mathbf{K}^{(\alpha, l)} L_{n}^{(\alpha)}(x)=0, n<l, \\
\mathbf{K}^{(\alpha, l)} L_{n}^{(\alpha)}(x)=L_{n}^{(\alpha)}(x), n \geq l,
\end{gathered}
$$

then for $n=l+1, l+2, \cdots$

$$
\begin{gathered}
\left(\mathbf{B}^{(\alpha, l)}-\left(\alpha_{n}^{(\alpha, l)}-\alpha_{l}\right) \mathbf{I}\right) L_{n}^{(\alpha)}(x)= \\
\frac{\left(\begin{array}{c}
n+\alpha \\
n-l
\end{array}\right)}{\left(\begin{array}{c}
l+\alpha \\
l
\end{array}\right)} \sum_{k=1}^{l+1}(-1)^{k}\left(\begin{array}{c}
n-k \\
l-k+1
\end{array}\right) k\left(L_{n-k}^{(\alpha+k)}(x)+L_{n-k-1}^{(\alpha+k+1)}(x)\right) .
\end{gathered}
$$

This last result is also valid for $l=0$ if in this case we take $\mathbf{A}^{(\alpha, l)}=\mathbf{B}^{(\alpha, l)}$. Using [10], Lemma 5, we obtain

$$
\begin{gathered}
(-1)^{i} \gamma_{i}(x ; \alpha, k)=L_{i-k}^{(-\alpha-i-1)}(-x) L_{k}^{(\alpha)}(x), \\
(-1)^{i} \kappa_{i}(x ; \alpha, l)=\sum_{j=l}^{i} L_{i-j}^{(-\alpha-i-1)}(-x) L_{j}^{(\alpha)}(x),
\end{gathered}
$$

$b_{i}(x ; \alpha, l)=0$ for $i \leq l$ and for $i \geq l+1$ we have

$$
\begin{gathered}
(-1)^{i} b_{i}(x ; \alpha, l)= \\
\sum_{j=l+1}^{i} L_{i-j}^{(-\alpha-i-1)}(-x) L_{j}^{(\alpha)}(x)\left[\frac{\Gamma(\alpha+1)}{(\Gamma(l+\alpha+1))^{2}} \sum_{k=l}^{j-1} \frac{\Gamma(k+\alpha+1) k !(j-k)}{((k-l) !)^{2}}\right] \\
+\sum_{j=l+1}^{i} L_{i-j}^{(-\alpha-i-1)}(-x) \frac{\left(\begin{array}{c}
j+\alpha \\
j-l
\end{array}\right)}{\left(\begin{array}{c}
l+\alpha \\
l
\end{array}\right)} \sum_{k=1}^{l+1}(-1)^{k}\left(\begin{array}{c}
j-k \\
l-k+1
\end{array}\right) k\left(L_{j-k}^{(\alpha+k)}(x)+L_{j-k-1}^{(\alpha+k+1)}(x)\right) .
\end{gathered}
$$




\section{Conclusions}

1. We note that for $\alpha$ a nonnegative integer

$$
\sum_{k=l}^{j-1} \frac{\Gamma(k+\alpha+1) k !(j-k)}{((k-l) !)^{2}}
$$

is a polynomial in $j$ of degree $\alpha+2 l+2$, divisible by $j(j-1) \cdots(j-l)$, and

$$
\left(\begin{array}{c}
j+\alpha \\
j-l
\end{array}\right)\left(\begin{array}{c}
j-k \\
l-k+1
\end{array}\right)
$$

is a polynomial in $j$ of degree $\leq \alpha+2 l+1-k$, divisible by $j(j-1) \cdots(j-l)$ if $j \leq l$. We have for nonnegative integer values of $\alpha$

$$
\begin{aligned}
& \frac{\Gamma(\alpha+1)}{(\Gamma(l+\alpha+1))^{2}} \sum_{k=l}^{j-1} \frac{\Gamma(k+\alpha+1) k !(j-k)}{((k-l) !)^{2}} \\
= & \frac{\Gamma(\alpha+1) j^{\alpha+2 l+2}}{(\Gamma(l+\alpha+1))^{2}(\alpha+2 l+1)(\alpha+2 l+2)}+\text { lower powers of } j .
\end{aligned}
$$

We now use formula (3) to see that for $\alpha$ a nonnegative integer the coefficients $b_{i}(x ; \alpha, l)$ vanish for $i>2 \alpha+4 l+4$, which was already known in the cases $l=0$ (see $[9]$ ) and $l=1$ (see [10]). Further

$$
b_{2 \alpha+4 l+4}(x ; \alpha, l)=\frac{\Gamma(\alpha+1)(-x)^{\alpha+2 l+2}}{(\Gamma(l+\alpha+1))^{2}(\alpha+2 l+1)(\alpha+2 l+2)} .
$$

2. By (3) in the case $s=0$ and $k=0$ it follows that

$$
\kappa_{i}(x ; \alpha, l)=(-1)^{i-1} \sum_{j=0}^{l-1} L_{i-j}^{(-\alpha-i-1)}(-x) L_{j}^{(\alpha)}(x) .
$$

If they occur the operators $\mathbf{J}^{(\alpha, k)}$ and $\mathbf{K}^{(\alpha, l)}$ are of infinite order. In the case that the eigenvalues $\alpha_{1}^{(\alpha, l)}, \alpha_{2}^{(\alpha, l)}, \cdots, \alpha_{l}^{(\alpha, l)}$ are all equal to zero, we have for $\alpha$ a nonnegative integer a differential operator of finite order $2 \alpha+4 l+4$ (if $M>0$ ) having the polynomials $L_{n}^{\alpha, M}(x, l)$ as eigenfunctions.

\section{ACKNOWLEDGMENT}

The author wishes to thank J. Koekoek for carefully reading the paper and correcting several errors.

\section{REFERENCES}

1. H. Bavinck: A direct approach to Koekoek's differential equation for generalized Laguerre polynomials. Acta Math. Hungar. 66 (3) (1995), 247-253. MR 96a:33009

2. H. Bavinck: Linear perturbations of differential or difference operators with polynomials as eigenfunctions. J. Comp. Appl. Math. 78 (1997), 179-195.

3. H. Bavinck: A new result for Laguerre polynomials. J. Physics A. 29 (1996), L277-L279. CMP 96:15

4. I.H. Jung, K.H. Kwon and G.J. Yoon: Differential equations of infinite order for Sobolev-type orthogonal polynomials. J. Comp. Appl. Math. 78 (1997), 277-293.

5. A.M. Krall: Orthogonal polynomials satisfying fourth order differential equations. Proc. Roy. Soc. Edinburgh, 87A (1981), 271-288. MR 82d:33021

6. A.M. Krall and L.L. Littlejohn: On the classification of differential equations having orthogonal polynomial solutions II. Ann. Mat. Pura Appl. (4) 149 (1987), 77-102. MR 89e:34017 
7. H.L. Krall: On orthogonal polynomials satisfying a certain fourth order differential equation. The Pennsylvania State College Studies, No. 6, The Pennsylvania State College, State College, Pa., 1940.

8. L.L. Littlejohn: An application of a new theorem on orthogonal polynomials and differential equations. Quaestiones Math. 10 (1986), 49-61. MR 87m:42021

9. J. Koekoek and R. Koekoek: On a differential equation for Koornwinder's generalized Laguerre polynomials. Proc. Amer. Math. Soc. 112, 1991, 1045-1054. MR 91j:33008

10. J. Koekoek, R. Koekoek and H. Bavinck: On differential equations for Sobolev-type Laguerre polynomials. TUDelft Fac. Techn. Math. \& Inf. Report 95-79. To appear in Trans. Amer. Math. Soc.

11. R. Koekoek: Generalizations of the classical Laguerre polynomials. J. Math. Anal. Appl. 153 (1990), 576-590. MR 92d:33016

12. R. Koekoek: Generalizations of the classical Laguerre polynomials and some q-analogues. Thesis Delft University of Technology 1990.

13. T.H. Koornwinder: Orthogonal polynomials with weight function $(1-x)^{\alpha}(1+x)^{\beta}$ $+M \delta(x+1)+N \delta(x-1)$. Canad. Math. Bull. 27(2), 1984, 205-214. MR 85i:33011

14. F. Marcellán and A. Ronveaux: On a class of polynomials orthogonal with respect to a Sobolev inner product. Indag. Math. N.S. 1 (1990), 451-464. MR 92f:42029

Delft University of Technology, Faculty of Technical Mathematics and Informatics, Mekelweg 4, 2628 CD Delft, The Netherlands

E-mail address: bavinck@twi.tudelft.nl 